Compliance to these PMs depends on the acceptability and adaptability of the general public to these PMs. However, there is a dearth of studies on this issue. Our study aimed to explore the perceptions and practices of COVID-19 related PMs among the general public in four districts of North India.

Methods The qualitative study was done in four administrative districts (Lucknow, Etawah, Patna and Darbhanga) in North India. All study districts had COVID-19 confirmed active cases at the time of data-collection. Participants in this study were healthy caregivers of under-five children, admitted for pneumonia, in a tertiary care hospital as part of ongoing study. Inclusion criteria was (i) child hospitalized in tertiary care hospital for pneumonia but presently discharged (ii) availability of telephone/mobile with caregiver and (iii) consent to participate. Audio in-depth interviews (IDIs) were conducted. Respondents were purposefully selected to represent both genders and two education groups (i) Below Primary Education Group ( $\leq 5$ years education) and (ii) Above Primary Education Group ( $>5$ years education). This ensured variability and heterogeneity in the population being studied. Data was managed using Atlast Ti. Conventional content analysis was used.

Results From July-Sep 2020, 60 IDIs were conducted; 36.6\% $(22 / 60)$ respondents were females and $26.6 \%(16 / 60)$ had below-primary education. Respondents concurred that most people in their society flouted the recommended PMs. The reasons for poor/non-compliance with PMs were: perceived poor susceptibility to illness, perceived less severity of COVID-19 and low perceived benefits of complying with PMs. Perceived severity of disease played a key role in shaping attitude and practices related to adherence of PMs. Most people believed that COVID-19 often had less severe outcome and is thus less fatal. The lack of direct experience with the disease also attenuated this belief and made them under-estimate the risk due to it. Social stigma associated with the disease caused many to hide the contagion and spread it rapidly. Respondents shared that many people in society 'bide' the disease due to fear of discrimination that was common for COVID-19 positive patients.

In addition, respondents opined that COVID-19 is less prevalent in rural areas and among the less educated population. Most respondents were aware of the recommended PMs and opined that these must be followed, however subjective norms, social norms and behavioural intentions negatively impacted compliance.

Conclusion Since there was poor community perception of susceptibility to COVID-19 as well as poor perceived severity, the community did not understand the benefits of applying to PMs. Therefore, future health communication strategies must take these into account to increase the possibility of success.

\section{P106 'IT'S REALLY STRANGE BECAUSE ALTHOUGH I'M WITH ALL MY FRIENDS, I'VE NEVER FELT SO LONELY AND I HAVE NO IDEA WHY': EXPLORING YOUNG PEOPLE'S EXPERIENCES OF THE COVID-19 PANDEMIC IN NORTH EAST ENGLAND: A QUALITATIVE DIARY-BASED STUDY}

Steph Scott*, Victoria J McGowan, Shelina Visram. Population Health Sciences Institute, Newcastle University, Newcastle-upon-Tyne, UK

10.1136/jech-2021-SSMabstracts. 192
Background Children and young people risk being 'disproportionately harmed' by the COVID-19 pandemic. Whilst an evolving body of literature focuses on the impact of the pandemic on the mental health and wellbeing of children and young people, less attention has been paid to the collection of qualitative, exploratory data. The aim of this study was to examine young people in North East England's experiences of COVID-19 and associated control measures.

Methods Flexible, qualitative diaries were collected with 31 young people aged 13-17 for six weeks between July and October 2020. Diary extracts were curated using Instagram Direct Messaging (DM), email and text messaging. At the end of the study, participants took part in a follow-up interview (conducted by telephone or Zoom), asking them to reflect on their diary entries.

Results Thematic analysis of diaries and interviews yielded three central themes: (1) impacts of social distancing upon mental health and emotional wellbeing; (2) impacts of the pandemic on education and school life; and (3) frustration, burden and responsibility.

Discussion These findings highlight acute mental health impacts (loneliness, isolation, anxiety) as well as longer-term repercussions from disrupted education (missed parts of curriculum, home schooling, cancelled exams, periods of isolation) on young people aged $13-17$ as a result of the COVID-19 pandemic.

\section{P107 WOMEN'S REACTIONS TO THE COVID-19 FOOD SYSTEM SHOCK AND INSIGHTS FOR STRATEGIES SUPPORTING HEALTHY PURCHASING AND DIETARY BEHAVIOURS: A QUALITATIVE STUDY} 1,2Janis Baird, ${ }^{1,2}$ Christina Vogel. 'Medical Research Council Lifecourse Epidemiology Unit, University of Southampton, Southampton, UK; ${ }^{2}$ National Institute for Health Research Biomedical Research Centre, University of Southampton, Southampton, UK; ${ }^{3}$ Global Health Research Institute, School of Human Development and Health, Faculty of Medicine, University of Southampton, Southampton, UK

\subsection{6/jech-2021-SSMabstracts.193}

Background The COVID-19 pandemic disrupted usual routines, including families' food acquisition and consumption patterns. This pandemic could have lasting effects on consumer behaviour and implications for future strategies to improve population diet. The aim of this study was to gain in-depth understanding of families lived experiences by i) examining the impact of disruption resulting from the pandemic on the food purchasing and eating behaviours of young women, and ii) identifying the insights these experiences bring to designing future healthy eating interventions.

Methods A cross-sectional sample of 34 customers aged 1845 years, from a UK discount supermarket chain completed semi-structured telephone interviews. Women were asked questions to understand their lived experiences of food shopping, cooking, and eating during the COVID-19 lockdown. Interviews were transcribed verbatim and analysed thematically using QSR NVIVO software. Six researchers were involved in developing the initial coding framework, double coding of eleven interview transcripts and refining the coding framework. 
Results Participants' median age was 35 years and 56\% were in paid employment. Findings show that participants displayed emotional responses to COVID-19 pandemic disruptions of their food purchasing and dietary behaviours. Anxiety was common, with many feeling anxious about not acquiring enough or preferred foods for their families; contracting COVID-19 while food shopping; or needing to balance food costs, meal/snack variety and health. Frustration was also widespread, particularly about those who stockpiled; the poor availability of products which caused challenging situations at family mealtimes; shoppers who did not respect social distancing rules; and queues/one-way systems that made food shopping longer or less successful. These negative emotions were balanced against feelings of empathy, altruism, and pleasure. Many participants were understanding of government and supermarket restrictions or took the risk of shopping in-store to leave delivery slots for vulnerable customers or to help neighbours. Several women enjoyed leaving the house/family to go food shopping but missed being able to browse for meal ideas; others liked having more family meals and time to cook healthier dishes together.

Conclusion Strategies to improve food purchasing and diet may be enhanced if positive emotions, like pleasure in families cooking and eating together, are targeted. As communicating the necessity for COVID-19 rules increased the public acceptance of these restrictions, a similar approach could be adopted for government policies to improve supermarket environments that may disrupt shoppers' routines but are necessary to improve shopping patterns.

\section{P108 CHARACTERISTICS OF LONG COVID: FINDINGS FROM A SOCIAL MEDIA SURVEY}

${ }^{1}$ Nida Ziauddeen*, ${ }^{2}$ Deepti Gurdasani, ${ }^{3}$ Margaret E O'Hara, ${ }^{3}$ Claire Hastie, 'Paul Roderick, ${ }^{4}$ Guiqing Yao, ${ }^{1,5,6}$ Nisreen A Alwan. ${ }^{1}$ School of Primary Care, Population Sciences and Medical Education, Faculty of Medicine, University of Southampton, Southampton, UK; ${ }^{2}$ William Harvey Research Institute, Queen Mary University of London, London, UK; ${ }^{3}$ Patient contributor, Long Covid Support; ${ }^{4}$ Department of Health Science, University of Leicester, Leicester, UK; ${ }^{5}$ NIHR Southampton Biomedical Research Centre, University of Southampton and University Hospital Southampton NHS Foundation Trust, Southampton, UK; ${ }^{6}$ NIHR Applied Research Collaboration Wessex, Southampton, UK

\subsection{6/jech-2021-SSMabstracts. 194}

Background Many people are not recovering for months after being infected with COVID-19. Long Covid (LC) is a major public health problem that needs defining, quantifying and describing. We aimed to explore and develop understanding of LC symptoms following mild/moderate COVID-19 infection and describe its impact on daily life.

Methods The survey was co-produced with people living with LC. Data was collected through an online social media survey mostly from online support groups using convenience nonprobability sampling. The criteria for inclusion were adults with lab-confirmed or suspected COVID-19 infection managed in the community (non-hospitalised) in the first two weeks of illness. We used agglomerative hierarchical clustering to identify specific symptom clusters, and their demographic, and functional correlates.

Results Data from 2550 participants with a median duration of illness of 7.7 months (interquartile range (IQR) 7.4-8.0) was analysed. The mean age was 46.5 years (standard deviation 11 years) with $82.8 \%$ females and $79.9 \%$ UK-based. $90 \%$ reported good, very good or excellent health prior to infection. Most participants described fluctuating (57.7\%) or relapsing LC symptoms (17.6\%). The most common initial symptoms that continued were exhaustion, headache, chest pressure/tightness and breathlessness. Cough, fever and chills were prevalent initial symptoms that became less so as the illness progressed. Cognitive dysfunction and palpitations became more common beyond the acute phase. $26.5 \%$ reported lab-confirmation of infection (NAAT or antibody). The biggest difference in symptoms between those who reported testing positive and those who did not was loss of smell/taste. Physical activity, stress and sleep disturbance were the most common symptom triggers. A third (32\%) reported they were unable to live alone without any assistance at six weeks from start of illness. 66.4\% reported taking time off sick, (median 60 days, IQR 20, 129). 37\% reported loss of income due to illness. Eighty four percent of participants reported ongoing symptoms affecting at least three organ systems. There were two main ongoing symptoms clusters; the majority cluster $(88.7 \%)$ exhibited mainly chest, cognitive symptoms and exhaustion, and the minority cluster (11.3\%) exhibited multi-system symptoms which had persisted from the start. The multi-system cluster reported more severe functional impact.

Conclusion This is an exploratory survey of LC characteristics. Whilst it is a non-representative sample, it highlights the heterogeneity of persistent symptoms, and the significant functional impact. To better characterise ongoing illness and prognosis, research is needed in a representative populationsample using standardised case definitions (to include those not lab-confirmed in the first pandemic wave).

\section{P109 MANAGING DISRUPTION AT A DISTANCE: UNEQUAL EXPERIENCES OF PEOPLE LIVING WITH LONG-TERM CONDITIONS DURING THE COVID-19 PANDEMIC}

${ }^{1}$ Stephanie Morris*, ${ }^{2}$ Josephine Wildman, ${ }^{2}$ Kate Gibson, ${ }^{2}$ Suzanne Moffatt, ${ }^{1}$ Tessa Pollard. ${ }^{1}$ Anthropology, Durham University, Durham, UK; ${ }^{2}$ Population Health Sciences Institute, Newcastle University, Newcastle-upon-Tyne, UK

\subsection{6/jech-2021-SSMabstracts. 195}

Rationale COVID-19 'lockdown' restrictions profoundly disrupted lives and often adversely affected health and wellbeing. Those who are clinically more vulnerable to COVID19 due to living with long term conditions (LTCs) were at most risk of negative impacts on their health and wellbeing, including in relation to their LTC self-management strategies. This study explores how people managed their LTCs, health and wellbeing under social distancing restrictions and self-isolation during the first wave of the COVID-19 pandemic, and examines why some people managed better than others.

Methods Telephone interviews were conducted between 11th May and 13th July 2020 with people $(n=44)$ aged 40 to 75 living in an urban and largely deprived area of North East England with one or more of eight LTCs, often including types 2 diabetes. Audio recorded interviews lasted 